\title{
On the extremality for Teichmüller mappings
}

\author{
By
}

HUANG XinZhong

\section{Introduction}

The universal Teichmüller space $T(1)$ is the totality of quasicircles with suitable normalization. This space $T(1)$ is universal in the sense that it contains the Teichmüller space of an arbitrary Riemann surface. There are several useful realization of $T(1)$. Among them the following Bers embedding method is now standard.

Consider $T(1)$ as the set of conformal mappings of the unit disk $E=$ $\{|z|<1\}$ onto domains surrounded by quasicircles, and take the Schwarzian derivatives of them. Then we can show that $T(1)$, which is now considered as the set of those Schwarzian derivatives, is a bounded domain in the Banach space $B_{2}$ consisting of all $\varphi$ holomorphic on $E$ with the norm

$$
\left\|\varphi \lambda^{-2}\right\|_{\infty}=\sup _{z \in E}\left|\varphi(z) \lambda(z)^{-2}\right|<\infty,
$$

where $\lambda^{2}(z)|d z|^{2}$ is the Poincare metric on $E$. For the relationship between quasicircles and Schwarzian, we refer to the works of Nehari [21], Ahlfors and Weill [2], Gehring and Pommerenke [8], and the author's recent work [15]. More detail reference for Teichmüller space, one can consult with, for instance, Lehto [19], Gardiner [6], Nag [20], and Imayoshi and Taniguchi [18].

The above representation tells us that for describing the whole universal Teichmüller space, we need only those holomorphic functions on $E$ with the order estimate

$$
|\varphi(z)|=O\left(\left(\frac{1}{1-|z|}\right)^{2}\right) .
$$

Because we know that the essential data for holomorphic quadratic differentials is the geometric structure of trajectories (or topologically, the foliation structure of them), we can assert that whole universal Teichmüller space can be controled by geometric structures of holomorphic functions $\varphi$ on $E$ satisfying the above order estimate.

This may lead us to the concept of the "Teichmüller model" of $T$ (1), which is the $L^{\infty}$-theory originated from Teichmüller, and whose main tool is a 
special kind of extremal quasiconformal mappings called Teichmüller mappings. (See the definition below.)

Teichmüller asserted that, in case of a compact Riemann surface, the Teichmüller space of it can be embedded in the space of holomorphic quadratic differentials by using, not the Schwarzian derivatives, but the Teichmüller mappings. A Teichmüller mapping is locally affine mapping in a sense, whose stretch direction can be controled rather directly by the geometric structure of the corresponding differential.

Even in case of the universal Teichmüller space, it is natural to consider that the complexity of the space can be represented by Teichmüller mappings and their geometric structures can be controled again by an order estimate of the corresponding differentials. But in general, a Teichmüller mapping need not be extremal (as Example 1 below shows), and even basic problems such as whether $T(1)$ can be represented in a space consisting of Teichmüller mappings still remain unsettled. We will investigate in this paper such basic problems and give several contributions.

Before stating our main result, we first give a historical survey of the problems and then discuss several fundamental conjectures.

Consider a quasiconformal mapping $f=f^{x}$ of the unit disk $E$ onto itself. The supperscript $\kappa$ denotes the complex dilatation of the mapping $f$. Such an $f$ induces a homeomorphism of the boundary $\partial E$ onto itself. Let $Q_{f}$ be the class of all quasiconformal self-mappings of $E$ with the same boundary values as $f$. In $Q_{f}$, there is a function $f_{0}$ with smallest maximal dilatation $K_{0}$. (Namely,

$$
K_{0}=\inf _{g \in Q f} K_{g} .
$$

Here $K_{f}$ denotes the maximal dilatation of $f$. Set $k_{0}=\left(K_{0}-1\right) /\left(K_{0}+1\right)$. Then this is equivalent to

$$
k_{0}=\inf _{g \in Q f} k_{g},
$$

where we set $k_{g}=\|\kappa\|_{\infty}$ for every $g=g^{\kappa}$.) Such a mapping $f_{0}$ is called extremal in the class $Q_{f}$, or more simply, extremal. An extremal mapping will be called uniquely extremal if there is only one extremal mapping in $Q_{f}$.

Next, a quasiconformal mapping $f(z)$ of $E$ is called a Teichmüller mapping if, for almost everywhere on $E$, the complex dilatation $\kappa$ of $f$ is of the form

$$
\kappa=k \frac{\bar{\varphi}}{|\varphi|}
$$

where $0<k<1$ and $\varphi$ is holomorphic on $E$.

Then a general question is the following: For a holomorphic function $\varphi$, when is the corresponding Teichmüller mapping extremal or uniquely extremal?

The origin of the problem goes back to

Grötzsch extremal problem ([9]). Show that if a schlicht and sing- 
le valued branch of $\int \sqrt{\varphi(z)} d z$ can be chosen which maps $E$ onto a rectangle, then any Teichmüller mapping corresponding to $\varphi$ is uniquely extremal.

However, a Teichmüller mapping need not be extremal, or may be extremal and yet not uniquely extremal as the following examples show.

Example 1. Let $\Phi$ map $E$ conformally onto the upper half plane and let $F(z)=K x+i y, K>1$. It follows that $f=\Phi^{-1} \circ F \circ \Phi$ is a Teichmüller mapping corresponding to $\varphi=\Phi^{2}$ which is not extremal, for if we set $G(z)=K z$ then $g=\Phi^{-1} \circ G \circ \Phi$ is conformal and agrees with $f$ on $\partial E$.

Example 2 (Strebel's chimney region [30]). Let $R=\{\operatorname{Im} z<0\} \cup$ $\{|\operatorname{Re} z|<1\}$, the mapping $F(z)=K x+i y$ minimizes the maximal dilatation in the class of all mappings of $R$ onto $S=F(R)$ that agree with $F$ on the boundary $\partial R$. If $\Phi$ and $\Psi$ are conformal mappings of $E$ onto $R$ and $S$ respectively, then $f=\Psi^{-1} \circ F \circ \Phi$ is a Teichmüller mapping corresponding to $\varphi=\Phi^{2}$ which is extremal in $Q_{f}$. However, if

$$
G(z)= \begin{cases}F(z) & \text { for } \operatorname{Im} z \geq 0 \\ K z & \text { for } \operatorname{Im} z<0\end{cases}
$$

the mapping $g=\Psi^{-1} \circ G \circ \Phi$ also has the maximal dilatation $K$ and $g$ agrees with $f$ on $\partial E$.

The problems of determining conditions on $\varphi$ in order that a corresponding Teichmüller mapping $f$ is extremal is seen to be one possible interpretation of the general problems stated by Teichmüller [32]. Main problems made by Teichmüller are to determine how regular the boundary correspondence should be in order to be continuable at all to a quasiconformal mapping and furthermore to be continuable to an extremal mapping which is a Teichmüller mapping.

The necessary and sufficient condition that the boundary correspondence be continuable to a quasiconformal mapping is given by Beurling and Ahlfors [3], but the second part of the problem is still unsolved, which we shall restate as Conjectures 1 and 2.

Actually, Teichmüller asked whether the condition $\iint_{E}|\varphi| d x d y<\infty$ is sufficient in order that a corresponding Teichmüller mapping is extremal. In 1962 , K. Strebel [30] succeeded in giving a partial answer to this question as well as to the case where the integral is infinite. In a later work [31] Strebel succeeded in giving affirmative answer to this question. However, Example 2 also shows that not every quasisymmetric boundary correspondence can be extended to a Teichmüller mapping with finite norm.

Now, in view of the above remarks, we are interested in the following

Extremality Conjecture 1. If $\varphi$ belongs to $B_{2}$, then every Teichmüller mapping corresponding to $\varphi$ is extremal. 
This conjecture has partially solved by many authors including Sethares [29], Reich and Strebel [28], and Hayman and Reich [12]. We will give in Theorem 1 a more general, but still partial answer for this conjecture. Also see Added in proof.

As for a possible characterization of $\varphi$, meromorphic in the closure of $E$, such that the corresponding Teichmüller mappings are extremal, Sethares [29] showed that, if $\varphi$ has at most double poles on $\partial E$, then a corresponding Teichmüller mapping $f$ is extremal. However, for every integer $n>2$, there exists such a $\varphi$, with an $n$-th order pole at $z=1$, for which a corresponding $f$ is not extremal. Based on these evidences, he made in [29] the following conjecture

Sethares conjecture [29]. For a Teichmüller mapping corresponding to holomorphic quadratic differential $\varphi$, which is meromorphic in a neighbourhood of $\bar{E}$, is extremal if and only if either (i) $\varphi$ has a double pole or (ii) $\varphi$ has no pole of order exceeding two.

The if-part of this conjecture was proved by Sethares himself, and the only-if-part is considered to be true, but have not been proved yet. A natural generalization of Sethares conjecture is Conjecture 1 and the following Conjecture 2. It is rather surprising that we can give a counterexample to Conjecture 2. See Examples 4 and 5 at the end of the section 3 .

Tameness Conjecture 2. Suppose that a Teichmüller mapping corresponding to holomorphic quadratic differential $\varphi$ is extremal. Then $\varphi$ belongs to $B_{2}$.

Finally, as for uniqueness of Teichmüller mappings, the following conjecture is modest, but still unsettled.

Uniqueness Conjecture 3. If $\varphi$ and $\phi$ belong to $B_{2}$, then Teichmüller mappings corresponding to $\varphi$ and $\phi$ concide with each other if only if $\varphi=\phi$ up to multiplication by a positive constant.

The uniqueness problem have been investigated by many mathematicians. There are several ways to attack this problem. First, by using Hamilton [11] and Reich-Strebel's [27] necessary and sufficient conditions for extremal quasiconformal mappings, Reich and Strebel proved the following:

Let $\kappa$ be an extremal complex dilatation for which no Hamilton sequence is degenerate. Then every weakly convergent Hamilton sequence tends in $L^{1}(E)$ to the unique holomorphic quadratic differential $\varphi$ and

$$
\kappa=\|\kappa\|_{\infty} \frac{\bar{\varphi}}{|\varphi|} .
$$

Next, considering the argument or the image domain for an extremal complex dilatation, Ortel and Smith [23], Huang [14], Huang and Taniguchi [17] also obtain the same assetion stated above.

Also by assuming various order estimates of $\varphi$, the uniqueness conjecture 
has been partially proved by Sethares [29], Hayman and Reich [12].

Acknowledgement. These results were obtained while I was a visiting member at the Department of Mathematics, Kyoto University. I wish to express my gratitude to my advisor, Professor M. Taniguchi for his inspiration and generous help. I benefit a lot from his encouragement, excellent guidence and his research attitude. I also wish to thank our colleague T. Sugawa and Professor H. Ohtake for several very useful discussions. At last, I like to thank the Department of Mathematics, Kyoto University for its support and hospitality.

\section{Preliminaries and main results}

Let $B(E)$ denote the class of functions $\varphi(z)$ holomorphic in $E$ which satisfy that

$$
\|\varphi(z)\|=\iint_{E}|\varphi(z)| d x d y<\infty, \quad z=x+i y
$$

For a Teichmüller mapping $f(z)$ of $E$ with the complex dilatation (1.5), a necessary and sufficient condition (see [11] and [28]) under which $f$ is an extremal mapping among the class of quasiconformal mappings of $E$ with the same boundary values as $f$ is that there exists a so-called Hamilton sequence, namely, a sequence $\left\{\varphi_{n}\right\}$ in $B(E)$, such that

$$
\lim _{n \rightarrow 0} \frac{\mid \iint_{E} \frac{\bar{\varphi}(z)}{|\varphi(z)| \varphi_{n}(z) d x d y \mid}}{\left\|\varphi_{n}(z)\right\|}=1 .
$$

If $\varphi(z) \in B(E)$, then $\varphi_{n}(z)=\varphi(z), n=1,2, \ldots$ constitutes a Hamilton sequence, so the problem to determine whether a Hamilton sequence exists or not is nontrivial only when $\|\varphi(z)\|=\infty$. Furthermore, if a Hamilton sequence $\left\{\varphi_{n}\right\}$ does exist, such a sequence can be realized in terms of a sequence of "polygonal" Teichmüller mappings $f_{n}$ which agree with $f$ at finitely many boundary points (see [28], Theorem 6). However, these $f_{n}$ 's are obtained by a highly nonconstructive process, hence the relationship between the properties of the corresponding sequence $\left\{\varphi_{n}\right\}$ and the properties of $\varphi$ is quite obscure. In [26] Reich considered the question whether a Hamilton sequence, if one does exist, can be obtained in a more direct manner. In particular, he considered the following: If $\left\{R_{n}\right\}$ is a sequence of numbers such that $0<R_{n}<1$ for every $n$ and $\lim _{n \rightarrow \infty} R_{n}=1$, does $\left\{\varphi\left(R_{n} z\right)\right\}$ constitute a Hamilton sequence? He showed

Theorem A. Suppose that $\varphi(z)$ is holomorphic in a neighbourhood of $\bar{E}$ except for a finite number of poles on $\{|z|=1\}$. Then

$$
\lim _{R \rightarrow 1} \frac{\left|\iint_{E} \frac{\bar{\varphi}(z)}{\varphi(z) \mid} \varphi(R z) d x d y\right|}{\|\varphi(R z)\|}=1
$$


if and only if $\varphi(z)$ has poles of at most order 2 on $\quad|| z \mid=1\}$.

From the work of Sethares [29], Theorem A is equivalent to the following

Theorem A'. Suppose that $\varphi(z)$ is holomorphic in a neighbourhood of $\bar{E}$ except for a finite number of poles on $\{|z|=1\}$. Then, $\left\{\varphi\left(R_{n} z\right)\right\}$ is a Hamilton sequence for any sequence $\left\{R_{n}\right\}$ as above if and only if the Teichmüller mapping with dilatation (1.5) is uniquely extremal.

In the work of Sethares [29], he obtained extremality and uniqueness theorems for Teichmüller mappings with $\|\varphi\|=\infty$ under assumptions on the growth of $\sup _{|z|=r}|\varphi(z)|$. On the other hand, in [28], Reich and Strebel obtained the following extremality theorem under a growth assumption on $I_{1}(r, \varphi)=\frac{1}{2 \pi} \int_{0}^{2 \pi}\left|\varphi\left(r e^{i \theta}\right)\right| d \theta$.

Theorem B. Suppose that $\varphi$ is holomorphic in E, and

$$
I_{1}(r, \varphi)=O\left(\frac{1}{1-r}\right), \quad r \rightarrow 1
$$

Then the Teichmüller mapping with dilatation (1.5) is extremal for its boundary values.

Later, in [12], Hayman and Reich showed

Theorem C. Suppose that $\varphi$ is holomorphic in $E$, and

$$
I_{1}(r, \varphi)=O\left(\frac{1}{1-r}\right), \quad r \rightarrow 1
$$

Then the Teichmüller mapping with dilatation (1.5) is uniquely extremal and $\left\{\varphi\left(R_{n} z\right)\right\}$ is a Hamilton sequence for any sequence $\left\{R_{n}\right\}$ as before.

The following example shows that extremality of $f$ is no longer implied if $(1-r)^{-1}$ in the right-hand of $(2.4)$ is replaced by $(1-r)^{-1-\delta}$, for any positive $\delta$.

Example. Let $\varphi(z)=\Psi^{\prime 2}(z)$, where $\zeta=\Psi(z)$ maps $E$ onto an angular domain, $|\arg \zeta|<\delta \pi / 4,0<\delta<2$. The horizontal stretching by a factor $K>1$ and subsequent conformal mapping of the streched angular domain onto the unit disk lead to a quasiconformal self-mapping of the unit disk with complex dilatation $\kappa(z)=k \bar{\varphi}(z) /|\varphi(z)|$, which is not extremal [30]. In this case, up to an irrelevant multiplicative constant,

$$
\varphi(z)=\frac{1}{(1-z)^{2+\delta}(1+z)^{2-\delta}}
$$

The order of $I_{1}(r, \varphi)$ for $(2.5)$ is the same as that of 


$$
I(r)=\int_{-\pi}^{\pi} \frac{1}{\mid 1-r e^{\left.i \theta\right|^{2+\delta}}} d \theta=\int_{-\pi}^{\pi} \frac{1}{\left(1-2 r \cos \theta+r^{2}\right)^{1+\frac{\delta}{2}}} d \theta .
$$

Since $\sin \frac{\theta}{2} \geq \frac{\theta}{\pi}$ for $0 \leq \frac{\theta}{2} \leq \frac{\pi}{2}$, we get that

$$
1-2 r \cos \theta+r^{2}=(1-r)^{2}+4 r \sin ^{2} \frac{\theta}{2} \geq(1-r)^{2}+\frac{4 r}{\pi^{2}} \theta^{2}
$$

Thus, for $r \geq \frac{1}{2}$,

$$
I(r) \leq \int_{-\pi}^{\pi} \frac{1}{\left((1-r)^{2}+2 \pi^{-2} \theta^{2}\right)^{1+\frac{\delta}{2}}} d \theta \leq \frac{1}{(1-r)^{1+\delta}} \int_{-\infty}^{\infty} \frac{1}{\left(1+2 \pi^{-2} t^{2}\right)^{1+\frac{\delta}{2}}} d t
$$

The last integral is convergent. Therefore,

$$
I_{1}(r, \varphi)=O\left(\frac{1}{(1-r)^{1+\delta}}\right), \quad r \rightarrow 1
$$

In this paper, we will prove an extremality theorem under growth assumptions on $I_{1}(r, \varphi)$ and on

$$
A(r, \varphi)=\frac{1}{\pi r^{2}} \int_{0}^{r} \int_{0}^{\pi}\left|\varphi\left(r e^{i \theta}\right)\right| r d r d \theta
$$

We know, from Hardy's convexity theorem (see [4], Theorem 1.5), that $I_{1}(r$, $\varphi)$ is a non-decreasing function of $r$, and

$$
A(r, \varphi) \leq I_{1}(r, \varphi) \leq \sup _{|z|=r}|\varphi(z)|
$$

Our results can be stated as follows.

Theorem 1. Suppose that $\varphi(z)$ is holomorphic in E,

$$
\lim _{r \rightarrow 1} \frac{\log \frac{1}{1-r}}{A(r, \varphi)}=0
$$

and that

$$
I_{1}(r, \varphi)=O\left(\frac{1}{1-r} \log \frac{1}{1-r}\right), \quad r \rightarrow 1 .
$$

Then the Teichmüller mapping with dilatation (1.5) is extremal for its boundary values, and there exists a sequence of numbers $\left\{\widetilde{R}_{n}\right\}$ such that $0<\widetilde{R}_{n}<1, \lim _{n \rightarrow \infty} \widetilde{R}$ ${ }_{n}=1$, and $\left\{\varphi\left(\widetilde{R_{n}} z\right)\right\}$ is a Hamilton sequence.

Sethares proved in [29] the following

Theorem D. For a holomorphic function $\varphi$, if $|\varphi(z)|=O(1 /(1-r))$ as $|z|=r \rightarrow 1$, then the Teichmüller mapping with dilatation (1.5) is extremal for its 
boundary values.

Corresponding to Theorem D, from Theorem 1, we obtain the following

Corollary 1. Suppose that $\varphi(z)$ is holomorphic in E, and that

$$
\sup _{|z|=r}|\varphi(z)|=O\left(\frac{1}{1-r} \log \frac{1}{1-r}\right), \quad r \rightarrow 1 \text {. }
$$

Suppose further that

$$
\lim _{r \rightarrow 1} \frac{\log \frac{1}{1-r}}{A(r, \varphi)}=0 .
$$

Then the Teichmüller mapping with dilatation (1.5) is extremal for its boundary values, and there exists a sequence of number $\left\{\widetilde{R}_{n}\right\}$ such that $0<\widetilde{R_{n}}<1, \lim _{n \rightarrow \infty} \widetilde{R}_{n}$ $=1$, and $\left\{\varphi\left(\widetilde{R}_{n} z\right)\right\}$ is a Hamilton sequence.

Also correponding to the Theorems $\mathrm{B}$ and $\mathrm{C}$, we have the following

Corollary 2. Suppose that $\varphi(z)$ is holomorphic in E, and that

$$
I_{1}(r, \varphi)=O\left(\frac{1}{1-r} \log \frac{1}{1-r}\right), \quad r \rightarrow 1 .
$$

If $\lim _{r \rightarrow 1}(1-r) I_{1}(r, \varphi)=\infty$, then the Teichmüller mapping with dilatation (1.5) is extremal and $\left\{\varphi\left(R_{n} z\right)\right\}$ is a Hamilton sequence for any $\left\{R_{n}\right\}$ such that $0<R_{n}$ $<1$ and $\lim _{n \rightarrow \infty} R_{n}=1$.

From these, we see that for the holomorphic functions $\varphi$ satisfying the assumptions made in Corollary 1 or 2, the Extremality Conjecture 1 is true.

Remark. In the case of $\lim _{r \rightarrow 1}(1-r) I_{1}(r, \varphi)=a \neq \infty$, the result had been proved in [28]. Thus corollary 2 treats the case which is not included in Theorem B.

\section{Proofs of Theorem 1 and Corollaries}

In order to prove Theorem 1, first we derive some relation between the mean growth of a holomorphic function and that of its derivative. Such results are related to the Theories of $H^{p}$ spaces and of univalent functions and interesting in themselves. The following Proposition is a counterpart of the results made by Duren [4], or by Reich [24], and the result made by Hallenbeck and MacGregor [10]. Some of other applications can also be seen from their works, hence are omitted here.

Proposition. Suppose that $\varphi(z)$ is holomorphic on $E, 0<p<\infty$ and $\beta>$ 0. Further suppose that 


$$
I_{p}^{\frac{1}{p}}(r, \varphi)=\left\{\frac{1}{2 \pi} \int_{0}^{2 \pi}\left|\varphi\left(r e^{i \theta}\right)\right|^{p} d \theta\right\}^{\frac{1}{p}}=O\left(\frac{1}{(1-r)^{\beta}} \log \frac{1}{1-r}\right), \quad r \rightarrow 1,
$$

then, for every positive integer $n$,

$$
I_{p}^{\frac{1}{p}}\left(r, \varphi^{(n)}\right)=\left\{\frac{1}{2 \pi} \int_{0}^{2 \pi}\left|\varphi^{(n)}\left(r e^{i \theta}\right)\right|^{p} d \theta\right\}^{\frac{1}{p}}=O\left(\frac{1}{(1-r)^{\beta+n}} \log \frac{1}{1-r}\right), r \rightarrow 1 .
$$

Proof of Proposition. First let $1 \leq p<\infty$, and assume that (2.8) holds. By the Cauchy formula

$$
\varphi^{\prime}\left(r e^{i \theta}\right)=\frac{1}{2 \pi i} \int_{|z|=R} \frac{\varphi(z)}{\left(z-r e^{i \theta}\right)^{2}} d z=\frac{R}{2 \pi} \int_{\psi=0}^{2 \pi} \frac{\varphi\left(R e^{i(\psi+\theta)}\right) e^{i(\psi-\theta)}}{\left(R e^{i \psi}-r\right)^{2}} d \psi
$$

where $R=\frac{(1+r)}{2}$. Suppose there exist $M>0$ such that $I_{p}^{\frac{1}{p}}(r, \varphi) \leq M \frac{1}{(1-r)^{\beta}}$ $\log \frac{1}{1-r}$. Minkowski’s inequality (in continuous form) then gives

$$
\begin{aligned}
I_{p}^{\frac{1}{p}}\left(r, \varphi^{\prime}\right) & \leq \frac{1}{2 \pi} \int_{\varphi=0}^{2 \pi} \frac{I_{p}^{\frac{1}{p}}(R, \varphi)}{R^{2}-2 R r \cos \varphi+r^{2}} d \phi \\
& =\frac{I_{p}^{p}(R, \varphi)}{R^{2}-r^{2}} \leq \frac{M}{(1-R)^{\beta}\left(R^{2}-r^{2}\right)} \log \frac{1}{1-R} \\
& =O\left(\frac{1}{(1-r)^{\beta+1}} \log \frac{1}{1-r}\right), \quad r \rightarrow 1 .
\end{aligned}
$$

Suppose now that $0<p<1$, and that

$$
I_{p}^{\frac{1}{p}}(r, \varphi) \leq \frac{M}{(1-r)^{\beta}} \log \frac{1}{1-r} .
$$

If $\varphi(z)$ has no zeros in $E$, let $F(z)=[\varphi(z)]^{p}$ is holomorphic and

$$
I_{1}(r, F) \leq \frac{M^{p}}{(1-r)^{\beta p}}\left(\log \frac{1}{1-r}\right)^{p} .
$$

With $R=\frac{1}{2}(1+r)$, this implies

$$
I_{1}\left(r, F^{\prime}\right) \leq \frac{I_{1}(R, F)}{R^{2}-r^{2}} \leq \frac{M_{1}}{(1-r)^{\beta p+1}}\left(\log \frac{2}{1-r}\right)^{p} .
$$

where $M_{1}>0$ is a constant. On the other hand, since

$$
\varphi^{\prime}(z) \leq \frac{1}{p}[F(z)]^{\frac{1}{p}-1} F^{\prime}(z),
$$

Hölder's inequality gives 


$$
\begin{aligned}
I_{p}^{\frac{1}{p}}\left(r, \varphi^{\prime}\right) & \leq \frac{1}{p} I_{1}^{\frac{1}{p}-1}(r, F) I_{1}\left(r, F^{\prime}\right) \\
& \leq \frac{1}{p}\left[\frac{M^{p}}{(1-r)^{\beta p}}\left(\log \frac{1}{1-r}\right)^{p}\right]^{\frac{1}{p}-1} \frac{M_{1}}{(1-r)^{\beta p+1}}\left(\log \frac{1}{1-r}\right)^{p} \\
& =O\left(\frac{1}{(1-r)^{\beta+1}} \log \frac{1}{1-r}\right), \quad r \rightarrow 1
\end{aligned}
$$

If $\varphi(z)$ has a zero, we need the following Lemma due to P.L. Duren [4].

Lemma. Any function $f \in H^{p}(0<p \leq \infty)$ can be expressed in the form $f(z)=f_{1}(z)+f_{2}(z)$, where $f_{1}$ and $f_{2}$ are nonvanishing $H^{p}$ functions such that $\left\|_{i}\right\|_{p}$ $\leq 2\|f\|_{p}$ for each $i$.

In this case, we fix $\mathrm{R}, 0<R<1$, and according to the above Lemma, we can write that

$$
\varphi(R z)=\varphi_{1}(z)+\varphi_{2}(z)
$$

where $\varphi_{1}$ and $\varphi_{2}$ are nonvanishing functions such that

$$
I_{p}^{\frac{1}{p}}\left(r, \varphi_{i}\right) \leq \frac{2 M}{(1-R)^{\beta}} \log \frac{1}{1-R}, \quad i=1,2 .
$$

Hence, by the same but more simple argument as in the first paragraph of this proof,

$$
I_{p}^{\frac{1}{p}}\left(r, \varphi_{i}^{\prime}\right) \leq\left(\frac{M_{2}}{(1-R)^{\beta}} \log \frac{1}{1-R}\right) \frac{1}{1-r}, \quad i=1,2,
$$

where $M_{2}>0$ is a suitable constant. Since

$$
R^{p}\left|\varphi^{\prime}(R z)\right|^{p} \leq 2^{p}\left[\left|\varphi_{1}^{\prime}(z)\right|^{p}+\left|\varphi_{2}^{\prime}(z)\right|^{p}\right]
$$

it follows that

$$
I_{p}^{\frac{1}{p}}\left(R^{2}, \varphi^{\prime}\right) \leq \frac{M_{3}}{(1-R)^{\beta+1}} \log \frac{1}{1-R}
$$

where $M_{3}>0$ is also a suitable constant. This easily gives (2.9). This proves Proposition with $n=1$.

Finally, applying the result with $n=1$ inductively, we can obtain the assertion for every $n$.

As a special case when $p=n=\beta=1$, the above Proposition turns out to be the following

Corollary 3. Suppose that $\varphi(z)$ is holomorphic on E, and

$$
I_{1}(r, \varphi)=O\left(\frac{1}{1-r} \log \frac{1}{1-r}\right), \quad r \rightarrow 1,
$$

then 


$$
I_{1}\left(r, \varphi^{\prime}\right)=O\left(\frac{1}{(1-r)^{2}} \log \frac{1}{1-r}\right), \quad r \rightarrow 1 .
$$

Proof of Theorem 1. In order to prove Theorem 1, first we note that, for every $R$ with $0<R<1$,

$$
\begin{aligned}
\alpha(R) & =\iint_{|z|<R}|\varphi(R z)-\varphi(z)| d x d y \leq \int_{0}^{R} r d r \int_{0}^{2 \pi} d \theta \int_{R r}^{r}\left|\varphi^{\prime}\left(t e^{i \theta}\right)\right| d t \\
& =2 \pi \int_{0}^{R} r d r \int_{R r}^{r} I_{1}\left(t, \varphi^{\prime}\right) d t .
\end{aligned}
$$

Hence, by using (2.11), the assumption implies that

$$
\begin{aligned}
\alpha(R) & \leq 2 M \pi \int_{0}^{R} r d r \int_{R r}^{r}\left(\frac{1}{(1-t)^{2}} \log \frac{1}{1-t}\right) d t \\
& \leq 2 M \pi \int_{0}^{R} \frac{r}{(1-r)^{2}} \log \frac{1}{1-r}\left(\int_{R r}^{r} d t\right) r d r \\
& \leq 2 M \pi(1-R) \int_{0}^{R} \frac{1}{(1-r)^{2}} \log \frac{1}{1-r} d r=2 M \pi(1-R) \int_{0}^{R} \log \frac{1}{1-r} d \frac{1}{1-r} \\
& =2 M \pi(1-R)\left[\frac{1}{1-R} \log \frac{1}{1-R}-\frac{R}{1-R}\right] \leq 2 M \pi \log \frac{1}{1-R},
\end{aligned}
$$

with a suitable M. Next, set

$$
\begin{aligned}
\beta(R) & =\iint_{R<|z|<1}|\varphi(R z)| d x d y=\int_{0}^{2 \pi} d \theta \int_{R}^{1}\left|\varphi\left(R t e^{i \theta}\right)\right| t d t \\
& =\frac{1}{R^{2}} \int_{0}^{2 \pi} d \theta \int_{R^{2}}^{R}\left|\varphi\left(u e^{i \theta}\right)\right| u d u=\frac{2 \pi}{R^{2}} \int_{R^{2}}^{R} r I_{1}(r, \varphi) d r .
\end{aligned}
$$

Again by the assumption, we obtain that

$$
\begin{aligned}
\beta(R) & \leq \frac{2 \pi}{R^{2}} \int_{R^{2}}^{R} \frac{M}{1-r} \log \frac{1}{1-r} d r=\frac{2 M \pi}{R^{2}} \int_{R^{2}}^{R} \log \frac{1}{1-r} d \log \frac{1}{1-r} \\
& =\frac{M \pi}{R^{2}} \log (1+R) \log \frac{1}{(1-R)^{2}(1+R)} \\
& \leq \frac{2 M \pi}{R^{2}} \log \frac{1}{1-R},
\end{aligned}
$$

with a suitable $M$.

Now, we prove that there exists a sequence of numbers $\left\{\widetilde{R}_{n}\right\}$ such that 0 $<\widetilde{R}_{n}<1, \lim _{n \rightarrow \infty} \widetilde{R}_{n}=1$, and

$$
\lim _{n \rightarrow \infty} \frac{\left|\iint_{E} \frac{\bar{\varphi}(z)}{\varphi(z) \mid} \varphi\left(\widetilde{R}_{n} z\right) d x d y\right|}{\left\|\varphi\left(\widetilde{R}_{n} z\right)\right\|}=1
$$

First, we can write 


$$
\begin{aligned}
& \frac{\iint_{E} \frac{\bar{\varphi}(z)}{|\varphi(z)|} \varphi(R z) d x d y}{\|\varphi(R z)\|}=R^{2}+R^{2} \frac{\iint_{|z|<R} \frac{\bar{\varphi}(z)}{\varphi(z) \mid}[\varphi(R z)-\varphi(z)] d x d y}{\iint_{|z|<R}|\varphi(z)| d x d y} \\
& +R^{2} \frac{\iint_{R<|z|<1} \frac{\bar{\varphi}(z)}{\varphi(z) \mid} \varphi(R z) d x d y}{\iint_{|z|<R}|\varphi(z)| d x d y} .
\end{aligned}
$$

By (2.12) and (2.13) we have

$$
0 \leq \frac{\left|\iint_{E} \frac{\bar{\varphi}(z)}{|\varphi(z)|}[\varphi(R z)-\varphi(z)] d x d y\right|}{\iint_{|z|<R}|\varphi(z)| d x d y} \leq \frac{\alpha(R)}{\pi R^{2} A(R, \varphi)} \leq \frac{2 M \pi \log \frac{1}{1-R}}{\pi R^{2} A(R, \varphi)}
$$

and

$$
0 \leq \frac{\left|\iint_{R<|z|<1} \frac{\bar{\varphi}(z)}{|\varphi(z)|} \varphi(R z) d x d y\right|}{\iint_{|z|<R}|\varphi(z)| d x d y} \leq \frac{\beta(R)}{\pi R^{2} A(R, \varphi)} \leq \frac{\frac{2 M \pi}{R^{2}} \log 1-R}{\pi R^{2} A(R, \varphi)}
$$

On the other hand, since $\underline{\lim }_{r \rightarrow 1} \frac{\log \frac{1}{1-r}}{A(r, \varphi)}=0$, there exists a sequence of numbers $\left\{\widetilde{R_{n}}\right\}$ such that $0<\widetilde{R_{n}}<1, \lim _{n \rightarrow \infty} \widetilde{R_{n}}=1$, and

$$
\lim _{n \rightarrow \infty} \frac{\log \frac{1}{1-\widetilde{R}_{n}}}{A\left(\tilde{R}_{n}, \varphi\right)}=0
$$

Therefore, combining (2.14) and (2.15), we deduce that

$$
\lim _{n \rightarrow \infty} \frac{\left|\iint_{E} \frac{\bar{\varphi}(z)}{\varphi(z)} \varphi\left(\widetilde{R}_{n} z\right) d x d y\right|}{\left\|\varphi\left(\widetilde{R}_{n} z\right)\right\|}=1
$$

Thus, the Teichmüller mapping with dilatation (1.5) is extremal and the sequence $\left\{\varphi\left(\widetilde{R}_{n} z\right)\right\}$ is a Hamilton sequence. The proof of the Theorem 1 is complete.

Since the proof of Corollary 1 can be easily deduced from Theorem 1, we will only prove Corollary 2.

Proof of Corollary 2. Suppose that $\varphi(z)$ satisfies the conditions of Corollary 2. Since $\lim _{r \rightarrow 1}(1-r) I_{1}(r, \varphi)=\infty$, using L'Hôpital's rule, we derive 


$$
\begin{aligned}
\lim _{r \rightarrow 1} \frac{\log \frac{1}{1-r}}{\pi r^{2} A(r, \varphi)} & =\lim _{r \rightarrow 1} \frac{\log \frac{1}{1-r}}{\int_{0}^{r} r\left(\int_{0}^{2 \pi}\left|\varphi\left(r e^{i \theta}\right)\right| d \theta\right) d r} \\
& =\lim _{r \rightarrow 1} \frac{1}{(1-r) r \int_{0}^{2 \pi}\left|\varphi\left(r e^{i \theta}\right)\right| d \theta}=0 .
\end{aligned}
$$

Thus by the proof of Theorem 1, we can see that the Teichmüller mapping with dilatation (1.5) is extremal and the sequence $\left\{\varphi\left(R_{n} z\right)\right\}$ is a Hamilton sequence for every $R_{n}$ as before. The proof of the Corollary 2 is complete.

Finally, we give an example to show that Theorem 1 really treats the case not included in Theorem B. It should be stressed that this example is also a counterexample for Tameness Conjecture 2.

Example 4. Set

$$
\varphi(z)=\frac{\log \frac{1}{1-z}}{(1-z)^{2}}
$$

on $E$. Then the Teichmüller mapping $f(z)$ with the dilatation $k \bar{\varphi}(z) /|\varphi(z)|, 0$ $<k<1$, is extremal for its boundary values.

In this case, we have

$$
\frac{1}{2 \pi} \int_{0}^{2 \pi}\left|\varphi\left(r e^{i \theta}\right)\right| d \theta=\frac{1}{2 \pi} \int_{0}^{2 \pi} \frac{\left|\log \frac{1}{1-r e^{i \theta}}\right|}{\left|1-r e^{i \theta}\right|^{2}} d \theta \leq C_{1} \frac{1}{1-r} \log \frac{1}{1-r}, \quad r \rightarrow 1 .
$$

On the other hand, because of $\lim _{r \rightarrow 1} \frac{\left|1-r e^{i(1-r)}\right|}{(1-r)}=\sqrt{2}$,

$$
\int_{0}^{2 \pi}\left|\varphi\left(r e^{i \theta}\right)\right| d \theta \geq \int_{0}^{(1-r)} \frac{\left|\log \frac{1}{1-r e^{i \theta}}\right|}{\left|1-r e^{i \theta}\right|^{2}} d \theta \geq C_{2}\left(\frac{1}{1-r} \log \frac{1}{1-r}\right), \quad r \rightarrow 1,
$$

where $C_{1}$ ans $C_{2}$ are positive constants. Therefore, there exists a constant $C_{3}>$ 0 such that

$$
A(r, \varphi)=\frac{1}{\pi r^{2}} \int_{0}^{r} \int_{0}^{2 \pi} \frac{1}{\left|1-r e^{i \theta}\right|^{2}}\left|\log \frac{1}{1-r e^{i \theta}}\right| r d r d \theta \geq C_{3}\left(\log \frac{1}{1-r}\right)^{2}, \quad r \rightarrow 1 .
$$

Thus $\lim _{r \rightarrow 1} \frac{\log \frac{1}{1-r}}{A(r, \varphi)}=0$, and hence, by Theorem 1, the Teichmüller mapping $f(z)$ with the dilatation $k \bar{\varphi}(z) /|\varphi(z)|$ is extremal for its boundary values. But $\varphi(z)$ does not satisfy the conditions in Theorem B.

Remark. Consider the conformal mapping 


$$
\Phi(z)=\left(\log \frac{2 e}{1-z}\right)^{\frac{3}{2}}
$$

of $E$. A simple computation shows that $f(z)$ is a Teichmüller mapping corresponding to

$$
\varphi(z)=\Phi^{\prime 2}(z)=\frac{1}{(1-z)^{2}}\left(\log \frac{2 e}{1-z}\right) .
$$

Then by Example 4, we conclude that

$$
f(z)=K \operatorname{Re} \Phi(z)+i \operatorname{Im} \Phi(z)
$$

is an extremal Teichmüller mapping for every positive $K>1$.

Example 5. By using Example 4, we can further construct a less obvious example, that is, a holomorphic function $\varphi(z)$ on $E$ which satisfies all of the following conditions.

i) Any Teichmüller mapping corresponding to $\varphi$ is extremal;

ii) $\varphi(z)$ has $\partial E$ as its natural boundary; (Namely, there are no $\Psi(z)$, holomorphic on a domain containing $E$ as a proper subset, which is coincident with $\varphi(z)$ on $E$.)

iii) For every $\zeta \in \partial E$ and every neighbourhood $V$ of $\zeta$ (in $\mathbf{C}$ ),

$$
\sup _{z \in V}(1-|z|)^{2}|\varphi(z)|=+\infty \text {. }
$$

First, fix a sequence $\left\{\alpha_{n}\right\}_{n=1}^{\infty}$, where every $\alpha_{n} \in \partial E$ and $\cup_{n=1}^{\infty}\left\{\alpha_{n}\right\}$ is dense in $\partial E$. Choose positive converging sequence $\left\{\varepsilon_{n}\right\}_{n=1}^{\infty}$ so that

$$
\varepsilon_{n} \geq \frac{2 C_{1} M_{2}}{C_{2}} \sum_{k=n+1}^{\infty} \varepsilon_{k}
$$

for every $n$, where $C_{1}, C_{2}$ are the same constants as in Example 4 and $M_{2}>1$ is a suitable positive constant. Let

$$
\varphi(z)=\sum_{k=1}^{\infty} \frac{\varepsilon_{k}}{\left(1-\bar{\alpha}_{k} z\right)^{2}} \log \frac{1}{1-\bar{\alpha}_{k} z}
$$

on $E$. Then $\varphi(z)$ satisfies the conditions. In fact, by the same estimate as in Example 4, it is easy to see that

$$
I_{1}(r, \varphi) \leq C_{1} \sum_{k=1}^{\infty} \varepsilon_{k} \frac{1}{1-r} \log \frac{1}{1-r} .
$$

And we have

$$
I_{1}(r, \varphi) \leq C_{2} \varepsilon_{1} \frac{1}{1-r} \log \frac{1}{1-r}
$$




$$
\begin{aligned}
& -C_{1} \sum_{k=2}^{\infty} \varepsilon_{k} \frac{1}{1-r} \log \frac{1}{1-r} \\
& \geq \frac{C_{2}}{2} \varepsilon_{1} \frac{1}{1-r} \log \frac{1}{1-r} .
\end{aligned}
$$

Hence

$$
\mathrm{A}(r, \varphi) \geq \frac{\varepsilon_{1}}{2} C_{3}\left(\log \frac{1}{1-r}\right)^{2}
$$

and as in Example 4, we conclude that i) holds.

Next, since ii) follows from iii), we will show that iii) holds. For this purpose, fix $\zeta \in \partial E$ and a neighborhood $V$ of $\zeta$ arbitrarity. Then there is an $\alpha_{N}$ in $V \cap \partial E$. Here we may assume that $\alpha_{j} \notin \bar{V} \cap \partial E$ for every $j<N$. Then

$$
\begin{aligned}
|\varphi(z)| & \geq\left|\frac{\varepsilon_{N}}{\left(1-\bar{\alpha}_{N} z\right)^{2}} \log \frac{1}{1-\bar{\alpha}_{N} z}\right| \\
& -\sum_{j<N}\left|\frac{\varepsilon_{j}}{\left(1-\bar{\alpha}_{j} z\right)^{2}} \log \frac{1}{1-\bar{\alpha}_{j} z}\right| \\
& -\sum_{j>N}\left|\frac{\varepsilon_{j}}{\left(1-\bar{\alpha}_{j} z\right)^{2}} \log \frac{1}{1-\bar{\alpha}_{j} z}\right|
\end{aligned}
$$

The second term in the right hand side of the above inequality is bounded on $V \cap E$ and we choose $M_{2}>1$, so that $\frac{C_{2} M_{1}}{C_{1} M_{2}}<1$, then

$$
\begin{aligned}
& \sum_{k>N}\left|\frac{\varepsilon_{k}}{\left(1-\bar{\alpha}_{k} z\right)^{2}} \log \frac{1}{1-\bar{\alpha}_{k} z}\right| \\
& \leq M_{1}\left(\sum_{k>N} \varepsilon_{k}\right) \frac{1}{(1-|z|)^{2}} \log ^{1-|z|} \mid \\
& \leq \frac{M_{1} C_{2} \varepsilon_{N}}{2 M_{2} C_{1}} \frac{1}{(1-|z|)^{2}} \log _{1-|z|} \mid \\
& \leq \frac{\varepsilon_{N}}{2} \frac{1}{(1-|z|)^{2}} \log \frac{1}{1-|z|} .
\end{aligned}
$$

Thus $(1-|z|)^{2}|\varphi(z)|$ tends to $+\infty$ when $z$ tends to $\partial E$ along the line $\arg z=$ $\left.\alpha_{N}\right\}$, which shows iii).

\section{Application of Theorem 1}

To describe the characteristic of extremal Teichmüller mappings from a variational point of view, it is natural to consider quasiconformal selfmappings of a domain which are equal to the identity on the boundary.

Let $Q_{I}$ be the class of quasiconformal mappings $f$ of the unit disk $E$ onto 
itself with $f\left(e^{i \theta}\right)=e^{i \theta}, 0 \leq \theta<2 \pi$. We write $\mathscr{F}_{0}$ the set of the complex dilatations of all $f \in Q_{I}$. Using the metric $|g(z)||d z|^{2}$ arising from a holomorphic quadratic differential $g(z) d z^{2}$ with finite norm, Reich and Strebel [27] derived a necessary integral condition for $\mu \in \mathscr{F}_{0}$, which is now called Main Inequality and plays a fundamental role in the theory of Teichmüller spaces. For instance, from this inequality one can easily derive a uniqueness theorem which states that a Teichmüller mapping $f$ which belongs to a quadratic differential $\varphi$ of finite norm is uniquely extremal with the class of mappings which coincide with $f$ on $\partial E$.

For other related results, also refer the works of Earle and Eells [5], Gehring [7], Reich [25] and Huang [13].

Because of the importance of the class of Teichmüller mappings it is desirable to estimate the growth of those quadratic differentials $\varphi$ for which there exists a $k>0$ such that $k \bar{\varphi} /|\varphi| \in \mathscr{F}_{0}$. In this case it is easy to know that $\|\varphi\|=\infty$. A simple example is the following

Example 6. Let $\Phi(z)=\frac{1+z}{1-z}$, which maps $E$ conformally onto the right half plane, and let $F(z)=K x+i y, K>1$. Then $f(z)=\Phi^{-1} \circ F \circ \Phi$ is a Teichmüller mapping corresponding to $\varphi=\Phi^{\prime 2}$. It is easy to see that $f(z) \epsilon$ $Q_{I}$. Furthermore, we have that $|\varphi|=O\left(\frac{1}{(1-r)^{4}}\right)$ and $I_{1}(r, \varphi)=O\left(\frac{1}{(1-r)^{3}}\right)$.

As Reich showed in [24] that, if $f \in Q_{I}$, and $\mu(z)=\frac{f_{\bar{z}}}{f_{z}}=k \frac{\bar{\varphi}(z)}{\mid \varphi(z)} \mid$, then by a theorem of Sethares [29], we have

$$
\varlimsup_{r \rightarrow 1}(1-r) \sup _{|z|=r}|\varphi(z)|=\infty,
$$

and by a theorem of Reich [24], we also have

$$
\varlimsup_{r \rightarrow 1}(1-r) I_{1}(r, \varphi)=\infty
$$

Using Theorem 1, we can derive that either the growing order for $I_{1}(r, \varphi)$ must be faster than $\frac{1}{1-r} \log \frac{1}{1-r}$, as is the case in example 6 , or $I_{1}(r, \varphi)$ and $A(r, \varphi)$ must satisfy some conditions. Namely, we get the following

Theorem 2. Suppose that $f \in Q_{I}$, and

$$
\mu(z)=\frac{f_{\dot{z}}}{f_{z}}=k \frac{\bar{\varphi}(z)}{|\varphi(z)|}
$$

where $\varphi(z)$ is holomorphic in E. Also suppose that

$$
I_{1}(r, \varphi)=O\left(\frac{1}{1-r} \log \frac{1}{1-r}\right), \quad r \rightarrow 1 .
$$


Then $A(r, \varphi)=O\left(\log \frac{1}{1-r}\right), r \rightarrow 1$, and (hence $\overline{\lim }_{r \rightarrow 1}(1-r) I_{1}(r, \varphi)=\infty$, but) $\underline{\lim }_{r \rightarrow 1}(1-r) I_{1}(r, \varphi)=a \neq \infty$.

Proof of Theorem 2. First we assume that

$$
\varlimsup_{r \rightarrow 1} \frac{A(r, \varphi)}{\log \frac{1}{1-r}}=\infty,
$$

which is equivalent to

$$
\lim _{r \rightarrow 1} \frac{\log \frac{1}{1-r}}{A(r, \varphi)}=0 .
$$

According to Theorem 1, the Teichmüller mapping $f$ with the complex dilatation $k \frac{\bar{\varphi}(z)}{|\varphi(z)|}$ is extremal for any positive $k(<1)$, but it is obvious that in the class of $Q_{I}$ the extremal mapping must be the conformal mapping $g(z)=z$. This contradiction proves that $A(r, \varphi)=O\left(\log \frac{1}{1-r}\right)$.

Next suppose that if $\underline{\lim }_{r \rightarrow 1}(1-r) I_{1}(r, \varphi)=\infty$. Then by the Corollary $2, f$ must be extremal, which is again impossible.

\author{
Department of Applied Mathematics \\ HUAQIAO UNIVERSITY \\ Quanzhou, Fujian, 362011, P.R.C.
}

\title{
References
}

[1] L. V. Ahlfors, On quasiconformal mappings, J. d'Analyse Math., 3 (1954), 1-58, 207-208.

[2] L. V. Ahlfors and G. Weill, A uniqueness theorem for Beltrami equations, Proc. Amer. Math. Soc., 13 (1962), 975-978.

[3] A. Beurling and L. Ahlfors, The boundary correspondence under quasiconformal mappings. Acta Math., 96 (1956), 125-142.

[4] P. L. Duren, Theory of $H^{p}$ Spaces, Academic Press, New York and London, 1970.

[5] C. J. Earle and J. Eells, Jr., On the differential geometry of Teichmüller spaces, J. d'Analyse Math., 19 (1976), 35-52.

[6] F. P. Gardiner, Teichmüller theory and quadratic differentials, John Wiley and Sons, Inc.. New York, 1987.

[7] F. W. Gehring, Quasiconformal mappings which hold the real axis pointwise fixed, Mathematical Essays Dedicated To A. J. MacIntyre, Ohio University Press (1970), 145-148.

[8] F. W. Gehring and Ch. Pommerenke, On the Nehari univalence criterion and quasicircles, Com. ment. Math. Helv., 59 (1984), 226-242.

[9] H. Grötzsch, Über möglichst konforme Abbildungen von schlichten Bereichen, Leipz. Ber., 84 (1932), 114-120.

[10] D. J. Hallenbeck and T. H. MacGregor, Integral estimates for the derivatives of functions in families defined through sobordination, Complex Variables, 3 (1984), 135-167. 
[11] R. S. Hamilton, Extremal quasiconformal mappings with prescribed boundary values, Trans. Amer. Math. Soc., 138 (1969), p.399-406.

[12] W. K. Hayman and E. Reich, On Teichmüller mappings of the disk, Complex Variables, 1 (1982), p.1-12.

[13] X. Z. Huang, A decomposition theorem of a class of plane quasiconformal mapping, Chinese J. of Contemporary Math., 9 (1988), 59-69.

[14] X. Z. Huang. The image domain of an extremal dilatation. Advances in Math. (China), 22 (1993), 435-440.

[15] X. Z. Huang, The Schwarzian derivative and quasicircles, to appear.

[16] X. Z. Huang, On conditions for Teichmüller mappings of the unit disk, to appear.

[17] X. Z. Huang and M. Taniguchi, On the contraction of the Teichmüller metrics, to appear.

[18] Y. Imayoshi and M. Taniguchi, An introduction to Teichmüller spaces, Springer-Verlag, 1992.

[19] O. Lehto, Univalent functions and Teichmüller spaces, Springer-Verlag, 1987.

[20] S. Nag. The complex analytic theory of Teichmüller spaces, Wiley interscience, 1988.

[21] Z. Nehari, The Schwarzian derivative and schlicht functions, Bull. Amer. Math. Soc., 55 (1949). 545-551.

[22] M. Ortel, Extremal quasiconformal mappings with angular complex dilatation, Indiana Univ. Math. J. 31 (1982), 435-447.

[23] M. Ortel and W. Smith. The argument of an extremal dilatation, Proc. Amer. Math. Soc., 104 (1988), 498-502.

[24] E. Reich, Extremality of some Teichmüller mappings with infinite norm, Proc. Romanian-Finnish Sem. Teichmüller Spaces and Quasiconformal Mappings, Brašov (1969), 245-254.

[25] E. Reich, On the decomposition of a class of plane quasiconformal mappings, Comment. Math. Helv., 53 (1978), 15-27.

[26] E. Reich, Construction of Hamiltion sequences for certain Teichmüller mappings, Proc. Amer. Math. Soc., 103 (1988), p.789-796.

[27] E. Reich and K. Strebel, On quasiconformal mappings which keep the boundary points fixed, Trans. Amer. Math. Soc., 138 (1969), 211-222.

[28] E. Reich and K. Strebel, Extremal quasiconformal mappings with given boundary values, Contributions to Analysis, a collections of Papers Dedicated to Lipman Bers, Academic Press. New York, 1974, p.375-391.

[29] G. C. Sethares, The extremal property of certain Teichmüller mappings, Comment. Math. Helv.. 43 (1968), p.98-119.

[30] K. Strebel, Zur Frage der Eindeutigkeit extremaler quasikonformer Abbildungen des Einheitskreises, Comment Math. Helv., 36 (1962), 306-323.

[31] K. Strebel, Zur Frage der Eindeutigkeit extremaler quasikonformer Abbildungen des Einheitskreises II , Comment. Math. Helv., 36 (1964), 77-89.

[32] O. Teichmüller, Extremale quasikonforme Abbildungen und quadratische Differentiale, Abh. Preuss. Akad. Wiss. Math-naturw. K1., 22 (1940), 1-197.

Added in proof: Extremality conjecture has counterexamples. See the following

[33] X. Z. Huang and H. Ohtake, A note on the extremality of Teichmüller mappings, to appear. 